\title{
Article
}

\section{Complex Numbers for Relativistic Operations}

\author{
Dmitry S. Kulyabov ${ }^{1,2 *(1)}$, Anna V. Korolkova ${ }^{1}$ (D) and Leonid A. Sevastianov ${ }^{1,2}$ (1) \\ 1 Peoples' Friendship University of Russia (RUDN University), 6 Miklukho-Maklaya St, Moscow, 117198, \\ Russian Federation; \{kulyabov-ds,korolkova-av,sevastianov-la\}@rudn.ru \\ 2 Joint Institute for Nuclear Research, 6 Joliot-Curie, Dubna, Moscow region, 141980, Russian Federation \\ * Correspondence: kulyabov-ds@rudn.ru
}

\begin{abstract}
When presenting special relativity, it is customary to single out the so-called paradoxes. One of these paradoxes is the formal occurrence of speeds exceeding the speed of light. An essential part of such paradoxes arises from the incompleteness of the relativistic calculus of velocities. In special relativity, the additive group is used for velocities. However, the use of only group operations imposes artificial restrictions on possible computations. Naive expansion to vector space is usually done by using non-relativistic operations. We propose to consider arithmetic operations in the special theory of relativity in the framework of the Cayley-Klein model for projective spaces. We show that such paradoxes do not arise in the framework of the proposed relativistic extension of algebraic operations.
\end{abstract}

Keywords: Cayley-Klein model; hypercomplex numbers; hyperbolic complex numbers; special relativity; superluminal motion

\section{Introduction}

Historically, special relativity arose to eliminate the inconsistencies of Maxwell's electrodynamics and Galileo's transformations. In this case, only group operations for speeds are traditionally considered: addition and subtraction of speeds. No other operations are used.

Thus, a situation arise when relativistic operations are used to add the velocities, and in other cases, in our opinion, non-relativistic operations are used. This leads to the emergence of the so-called paradoxes.

The authors suggest that it is necessary to extend the relativistic operations with velocities to algebra. It is assumed that this extension will remove some of the so-called paradoxes of the special theory of relativity.

Several attempts have been made to describe relativistic operations consistently. Most of these systems are quite complex [1-3]. The authors propose not to create a new algebra, but to use in this role the systems of complex numbers based on the Cayley-Klein model [4-9].

\subsection{Article structure}

In the paragraph 1.2 the basic notation and conventions used in the paper are provided. The section 2 describes the model structure of special relativity. In the section 3 , the authors substantiate the choice of the mathematical apparatus for the implementation of the model. In the section 4, several so-called paradoxes of special relativity are given, which are still quite common in popular science, and even in the scientific community. Section 9 shows that when using the formalism described in the paper, no paradoxes arise. Section 5 describes the projective Cayley-Klein model. Further, in the section 6 , complex numbers are introduced that realize the spaces of the Cayley-Klein model. The information contained in these two paragraphs is widely known, and the sections are more of a reference in nature. Section 7 introduces a technique for implementing relativistic operations based on hyperbolic complex numbers. It is necessary to draw the 
reader's attention that this implementation describes only collinear movements in one spatial dimension. This is done on purpose to simplify the perception of the proposed formalism. In section 8 , the basic relativistic operations are written out explicitly.

\subsection{Notations and conventions}

1. Designation of types of measure definition:

- elliptic measure is denoted by the symbol "-";

- parabolic measure is denoted by the symbol " 0 ";

- hyperbolic measure is denoted by the symbol " + ".

2. Designation of distances between points:

- $\quad-\mathrm{d}_{A B}$ : elliptic distance between points $A$ and $B$;

- $\quad{ }^{0} \mathrm{~d}_{A B}$ : parabolic distance between points $A$ and $B$;

- $\quad{ }^{+} \mathrm{d}_{A B}$ : hyperbolic distance between points $A$ and $B$.

3. Designation of measure of angles:

- $\quad{ }^{-} \delta_{a b}$ : elliptic measure of the angle between straight lines $a$ and $b$;

- $\quad{ }^{0} \delta_{A B}$ : parabolic measure of the angle between straight lines $a$ and $b$;

- $\quad{ }^{+} \delta_{A B}$ : hyperbolic measure of the angle between straight lines $a$ and $b$.

4. Imaginary units:

- i: elliptic imaginary unit;

- $\quad \varepsilon$ : parabolic imaginary unit;

- $\quad$ e: hyperbolic imaginary unit;

\section{Model structure of special relativity}

The general model structure of the theory consists of theoretical and operational parts (Fig. 1). The theoretical part consists of a model layer and an implementation layer. Special relativity is based on two postulates [10]:

1. Einstein's principle of relativity: the laws of nature are the same in all reference frames moving rectilinearly and uniformly relative to each other.

2. The principle of the constancy of the speed of light: the speed of light in a vacuum is the same in all reference frames moving rectilinearly and uniformly relative to each other [11].

In order for Maxwell's electrodynamics to comply with the principle Einstein's relativity, it was necessary to replace Galileo's transformations with Lorentz's transformations.

When implementing the mathematical apparatus of special relativity, the boundary between the theoretical part and the operational measuring part was shifted towards the theoretical part. All theoretical calculations are carried out in the same form in which we get the results. ${ }^{1}$ On the one hand, this simplifies specific calculations, on the other hand, it makes it difficult to understand the structure of the theoretical part (which is why there are so many fighters against the special theory of relativity). In addition, it complicates the development of this theory. The relations derived by the classics of the special theory of relativity are widely used. But the derivation of new relationships is somewhat difficult. It seems that it is with this that a large number of so-called paradoxes of the theory of relativity are connected.

We believe that it is necessary to strictly distinguish between the theoretical and operational parts. For this, it is proposed to use for theoretical calculations the mathematical formalism that implements the geometric structure of the space of special relativity, and only at the end to translate it into the required form through the measuring operational part.

1 As an opposite approach, we may mention the quantum theory, where the theoretical part is extremely complex and completely different from the results obtained as a result of measurements. 


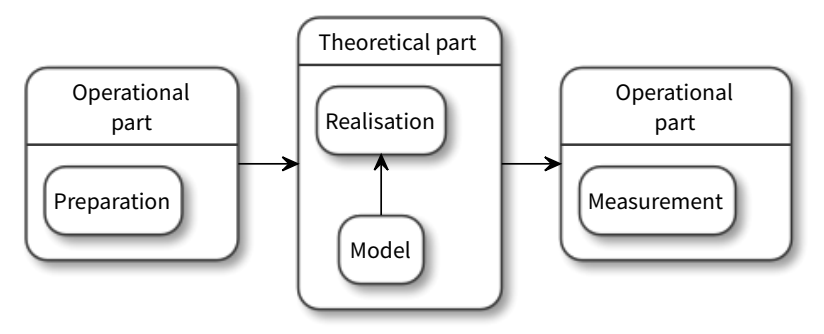

Figure 1. Model structure of the theory

\section{Justification of the choice of implementation}

The apparatus of hypercomplex numbers is chosen as the mathematical apparatus for implementing the model of special relativity.

- For implementation, it is preferable to use not a general formalism (describing an arbitrary structure), but a formalism specific to a particular geometry [12].

- Since we propose to use the Cayley-Klein space model, it seems quite reasonable to use one of the standard implementations of operations in Cayley-Klein spaces.

- The Cayley-Klein classification defines measures separately for lengths, angles, etc. It is preferable to be able to set the mathematical apparatus, taking into account the measures of the different elements of space. ${ }^{2}$

- The apparatus of complex numbers may be extended in a standard way to describe large dimensions (Cayley-Dickson procedure [13]).

- The formalisms of complex numbers and quaternions are quite familiar to the vast majority of scientists. ${ }^{3}$

- There are computer implementations for working with generalized complex numbers [9].

Based on the above considerations, we have chosen the formalism of hypercomplex numbers for the implementation of relativistic operations.

\section{Special relativity paradoxes}

In the literature on the special theory of relativity, relations regularly arise in which the calculated speed is higher than the speed of light $(v>c)$. All of these examples can be spotted in several classes. In this section, we list the most famous examples of paradoxes arising from the use of multiplication or division by a number.

\subsection{Oblique incidence of light}

The simplest model of superluminal motion can be an oblique incidence of plane waves on a certain flat interface between the media (also called a screen) [14-17]. Let $\varphi$ be the angle of incidence of the wave on the screen, that is, the angle between the wave vector and the normal to the screen. Then the light spot on the screen moves across this screen with the speed:

$$
v=\frac{c}{n \sin \varphi} .
$$

Here $n$ is the refractive index of the medium in which the light pulse propagates (the medium above the screen). Since $\sin \varphi \leqslant 1$, then the speed of the light spot with a decrease in the angle of incidence $\varphi$ can be made greater than the speed of light $c$. When considering the case of wave propagation in a vacuum, this becomes most obvious:

$$
v=\frac{c}{\sin \varphi}
$$

2 For example, when constructing the Minkowski space, only boosts were taken into account, that is, measures of angles. The measure definitions of lengths were simply borrowed from Euclidean space. But at the same time, it is good to be able to combine different definitions.

3 This point is probably the most important for us. It is not difficult to create a complex and cumbersome formalism. But nobody will use it. 
The velocity $v$ can be arbitrarily large since as the light pulse tends to normal incidence $(\varphi \rightarrow 0)$, then the velocity tends to infinity $(v \rightarrow \infty)$.

\subsection{Phase velocity}

The phase velocity of a wave is the speed of propagation of the surface of a constant phase along a given direction. The phase velocity in the direction of the wave vector is set as follows:

$$
v_{p}=\frac{\omega}{k}=: v_{p}(0),
$$

where $\omega$ is the circular frequency, $k$ is the wavenumber. In a vacuum for an electromagnetic wave, the phase velocity along the vector is equal to the speed of light $c$.

When deviating from the wave vector by an angle $\varphi$, the phase velocity will be equal to:

$$
v_{p}(\varphi)=\frac{v_{p}(0)}{\cos \varphi} .
$$

From the equation (2), it is seen that the phase speed can be greater than the speed of light [18].

\section{Cayley-Klein model}

Spatial measurements are reduced to two main tasks: to determine the distance between two points and to determine the angle between two intersecting lines. These tasks can be characterized as a problem of measure in projective geometry. According to the Cayley-Klein scheme $[4,19]$ there are three types of measurement: elliptic, parabolic, and hyperbolic. Consider what these measurements are for lengths and angles.

Let us denote the elliptic measure by the symbol " - ", the parabolic measure by the symbol " 0 ", the hyperbolic measure by the symbol " + ". 4

\subsection{Lengths measuring}

According to the Cayley-Klein scheme, there are three geometries on the straight line $o$ that define the measure of lengths: elliptic, parabolic, and hyperbolic geometries.

\subsubsection{Elliptic measurement of lengths}

The elliptic measure of lengths is defined on the line $o$ as follows (see Fig. 2). We define outside $o$ some point $Q$ and assume that the distance between the points $A$ and $B$ of the straight line $o$ is equal to the angle $\angle A Q B$ :

$$
-\mathrm{d}_{A B}=\angle A Q B .
$$

For consecutive points $A, B, C$ (see Fig. 2) we have the following equality:

$$
{ }^{-} \mathrm{d}_{A C}+{ }^{-} \mathrm{d}_{C B}={ }^{-} \mathrm{d}_{A B} .
$$

The elliptic geometry may serve as an example of an elliptic measure of lengths.

\subsubsection{Parabolic measurement of lengths}

Parabolic lengths are defined on the line $o$ as follows (see Fig. 3). The unit of length $O E$ is fixed on the straight line. The distance between the points $A$ and $B$ of the straight line $o$ is defined as

$$
{ }^{0} \mathrm{~d}_{A B}=\frac{A B}{O E} .
$$

For consecutive points $A, B, C$ (see Fig. 3) the following equality is true:

$$
{ }^{0} \mathrm{~d}_{A C}+{ }^{0} \mathrm{~d}_{C B}={ }^{0} \mathrm{~d}_{A B} .
$$

\footnotetext{
4 These designations are based on the designations for the complex numbers corresponding to each measure, see section 6.
} 


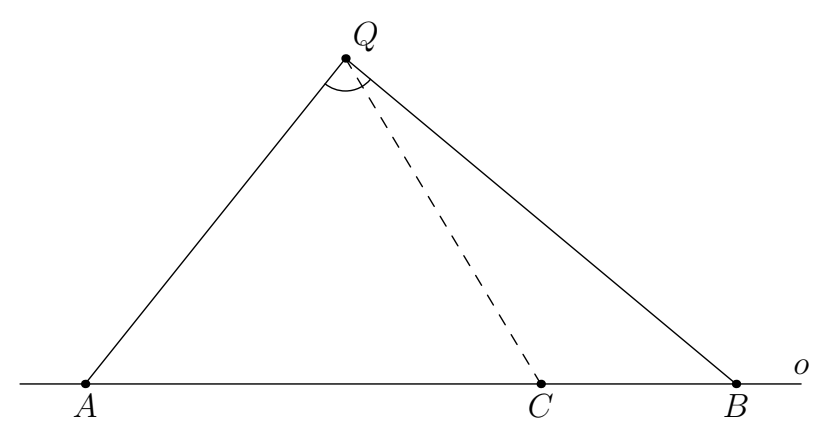

Figure 2. Elliptic measurement of lengths

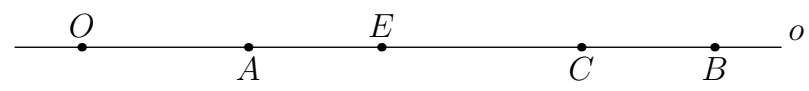

Figure 3. The parabolic measurement of lengths

An example of a parabolic measure of lengths is the geometry of Euclidean familiar to us.

\subsubsection{Hyperbolic measurement of lengths}

The hyperbolic measure is defined on the line $o$ as follows. Between two points $I$ and $J$ of the line $o$, two points $A$ and $B$ of this line are specified (see Fig. 4 ). Then the hyperbolic distance will have the form:

$$
{ }^{+} \mathrm{d}_{A B}=\varkappa \ln \frac{A I / A J}{B I / B J} .
$$

The $\varkappa$ coefficient specifies the distance units (and the base of the logarithm). The angles are considered to be directed. The entire hyperbolic line is represented by the segment IJ.

For consecutive points $A, B, C$ (see Fig. 4) the following equality is true:

$$
{ }^{+} \mathrm{d}_{A C}+{ }^{+} \mathrm{d}_{C B}={ }^{+} \mathrm{d}_{A B} .
$$

An example of a hyperbolic measure of lengths is the geometry of Lobachevsky.

\subsection{Angles measurement}

Similar to the three geometries on the straight line $o$, three geometries are introduced in the bunch of straight lines with the center $O$, that is, three systems of measures for determining the angles between the straight lines of the bunch are given.

\subsubsection{Elliptic measurement of angles}

The elliptic measure of angles in a bunch centered at $O$ is defined as follows (see Fig. 5):

$$
{ }^{-} \delta_{a b}=\angle a O b \text {. }
$$

For the straight lines of the bunch $a, b, c$ (see Fig. 5) the following equality is true:

$$
{ }^{-} \delta_{a c}+{ }^{-} \delta_{c b}={ }^{-} \delta_{a b}
$$

An example of an elliptic measure of angles is the geometry of Euclid, which is familiar to us.

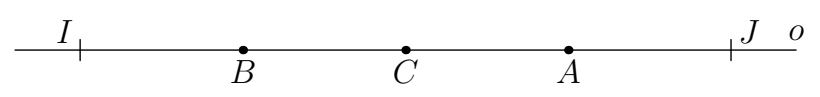

Figure 4. Hyperbolic measurement of lengths 


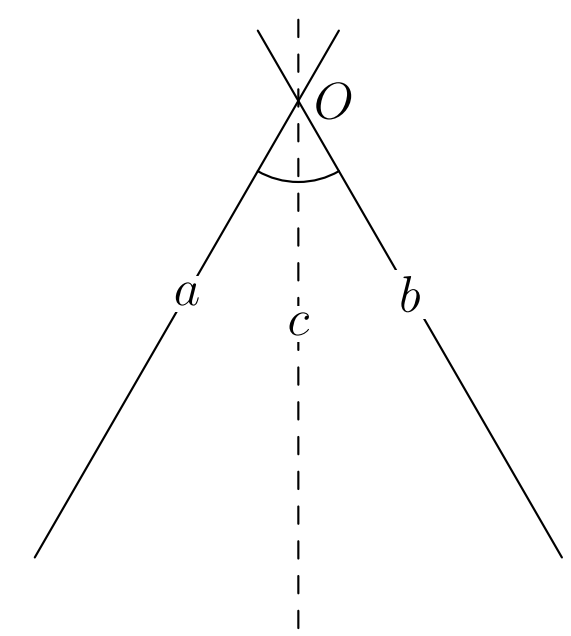

Figure 5. Elliptic measurement of angles

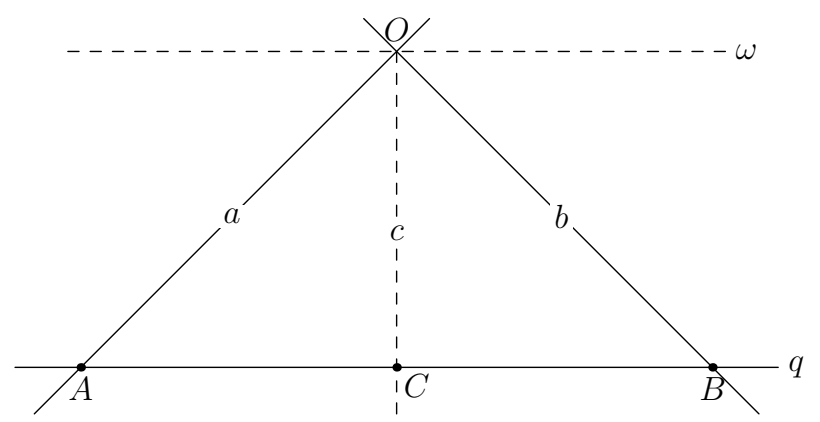

Figure 6. Parabolic measurement of angles

\subsubsection{Parabolic measurement of angles}

The parabolic measure of angles in a bunch with center $O$ is defined as follows (see Fig. 6). We fix the straight line $q$ not passing through the point $O$ and set the distance between the angles:

$$
{ }^{0} \delta_{a b}=A B
$$

Here the points $A$ and $B$ are the intersection points of the lines $a$ and $b$ with $q$. In addition, note that the bunch of lines contains a unique line $\omega \| q$, forming an infinitely large angle with any other straight line.

Obviously, from (3) and (5) we may write:

$$
{ }^{0} \delta_{a b}={ }^{0} \mathrm{~d}_{a b} .
$$

For the straight lines of the bunch $a, b, c$ (see Fig. 6) we have the following equality:

$$
{ }^{0} \delta_{a c}+{ }^{0} \delta_{c b}={ }^{0} \delta_{a b} .
$$

An example of a parabolic measure of angles is Galileo's geometry. ${ }^{5}$ 


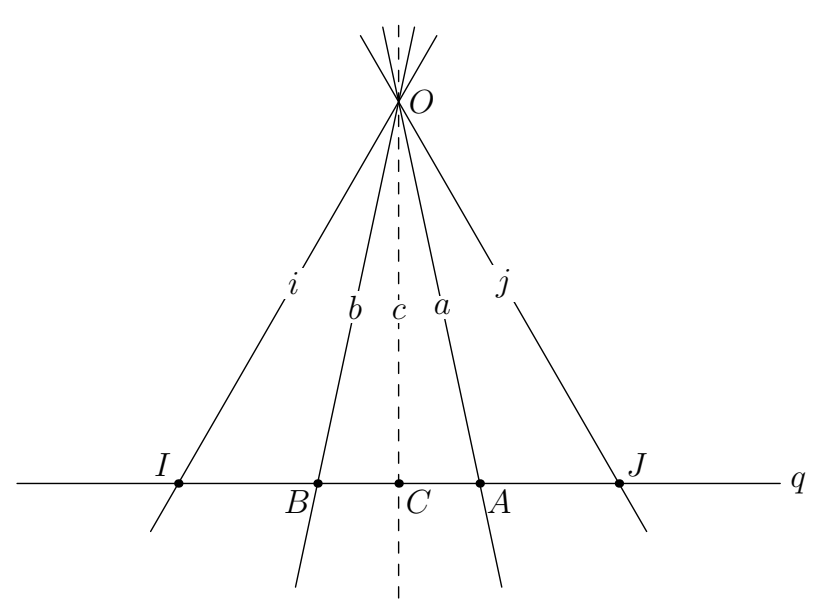

Figure 7. Hyperbolic measurement of angles

Table 1: Cayley-Klein geometries on a plane

\begin{tabular}{|c|l|l|l|}
\hline Lengths & \multicolumn{1}{|c|}{ Angles } & \multicolumn{1}{c|}{0} & \multicolumn{1}{c|}{+} \\
\hline- & Elliptic (Riemann) & co-Euclidean & $\begin{array}{l}\text { co-Hyperbolic (anti } \\
\text { de-Sitter) }\end{array}$ \\
\hline 0 & Euclidean & Galilean & Minkowski \\
\hline+ & $\begin{array}{l}\text { Hyperbolic } \\
\text { (Lobachevsky) }\end{array}$ & co-Minkowski & $\begin{array}{l}\text { Doubly hyperbolic } \\
\text { (de-Sitter) }\end{array}$ \\
\hline
\end{tabular}

\subsubsection{Hyperbolic measurement of angles}

The hyperbolic measure of angles in a bundle with center $O$ is defined as follows (see Fig. 7). We fix two straight lines $i$ and $j$ of the bundle and for any two other straight lines $a$ and $b$ we set the angular distance between them:

$$
{ }^{+} \delta_{a b}=\varkappa \ln \frac{\sin \angle a i / \sin \angle a j}{\sin \angle b i / \sin \angle b j} .
$$

The $\varkappa$ coefficient specifies the distance units (and the base of the logarithm). The angles are considered to be directed.

Since the double ratio from the equation (7) of four straight lines of the bunch is equal to the double ratio from the equation (4) of the four points of intersection of these lines with an arbitrary fifth straight line $q$ not passing through the center of the bunch, we have equality

$$
{ }^{+} \delta_{a b}={ }^{+} \mathrm{d}_{A B} .
$$

Here $A$ and $B$ are the intersection points of an arbitrarily fixed line $q$ with lines $a$ and $b$ (see Fig. 7). Each of the straight lines $i$ and $j$ forms an infinitely large angle with any other straight line.

For the straight lines of the bunch $a, b, c$ (see Fig. 7) the following equality is true:

$$
{ }^{+} \delta_{a c}+{ }^{+} \delta_{c b}={ }^{+} \delta_{a b} .
$$

The geometry of Minkowski may serve as an example of a hyperbolic measure of angles.

\subsection{Cayley-Klein projective geometries}

According to the Cayley-Klein scheme, nine projective geometries are defined on the plane (see Table 1) [5]. It is determined by fixing the measurement of lengths and angles. 


\section{Cayley-Klein model and complex numbers}

Points in the Euclidean plane can be identified with complex numbers by associating a point with Cartesian rectangular coordinates $(x, y)$ or with polar coordinates $(r, \varphi)$ to a complex number:

$$
z=x+\mathrm{i} y=r \exp \{\mathrm{i} \varphi\},
$$

where $\mathrm{i}^{2}=-1$. The quantities $x$ and $y$ are called, respectively, the real and imaginary parts of the number $z$ :

$$
x=\operatorname{Re}\{z\}, \quad y=\operatorname{Im}\{z\} .
$$

However, you can give a general definition of complex numbers. Let's set a quadratic equation in the form:

$$
z^{2}+p z+q=0
$$

The determinant of the quadratic equation (8) will have the form:

$$
\Delta=p^{2}-4 p q .
$$

Depending on the sign of the determinant, we can obtain the following systems of complex numbers ${ }^{6}[6]$ :

- $\Delta<0, z=a+\mathrm{i} b, \mathrm{i}^{2}=-1$ : elliptic complex numbers (ordinary complex numbers);

- $\Delta=0, z=a+\varepsilon b, \varepsilon^{2}=0$ : parabolic complex numbers (dual numbers) [6];

- $\Delta>0, z=a+\mathrm{e} b, \mathrm{e}^{2}=1$ : hyperbolic complex numbers (split complex number, hyperbolic number, perplex number [21,22], double number [6]).

Only ordinary complex numbers have a field structure. Dual and double complex numbers have a ring structure because they contain nontrivial zero divisors.

Here are the basic representations and equations for complex numbers. ${ }^{7}$ We will denote elliptic complex numbers by the symbol " -", parabolic complex numbers by the symbol " 0 ", and hyperbolic complex numbers by the symbol " + ".

6.1. Basic representations of complex numbers

6.1.1. Algebraic representation

Algebraic representation is the most common representation for complex numbers. This representation is most convenient for recording additive operations (addition, subtraction).

$$
\begin{aligned}
& z=a+\mathrm{i} b \\
& z=a+\varepsilon b \\
& z=a+\mathrm{e} b
\end{aligned}
$$

\subsubsection{Trigonometric representation}

In trigonometric representation, the real and imaginary parts of the complex number $z$ are expressed in terms of the modulus $r=|z|$ and the $\operatorname{argument} \varphi=\operatorname{Arg} z$ of the

\footnotetext{
6 Note that in the literature there is also a reverse name for complex numbers:

- $\Delta<0$ : hyperbolic complex numbers;

- $\Delta=0$ : parabolic complex numbers (dual numbers);

- $\Delta>0$ : elliptic complex numbers.
}

7 These formulas are well known, but we found it possible to cite them for the convenience of the reader. 
given complex number. This representation is most convenient for writing multiplicative operations (multiplication, division, exponentiation, root extraction).

$$
\begin{gathered}
z=r(\cos \varphi+\mathrm{i} \sin \varphi) \\
z=r(1+\varepsilon \varphi) ; \\
z= \begin{cases}r(\cosh \varphi+\mathrm{e} \sinh \varphi), & |a|>|b|, \\
0, & |a|=|b|, \\
r(\sinh \varphi+\operatorname{e} \cosh \varphi), & |a|<|b| .\end{cases}
\end{gathered}
$$

We also need to define the modulus and argument of a complex number.

Complex number module

The value $r=|z|$ is called the modulus of the complex number $z$ :

$$
\begin{gathered}
r=\sqrt{a^{2}+b^{2}} ; \\
r=a ; \\
r= \begin{cases}\sqrt{a^{2}-b^{2}}, & |a|>|b|, \\
0, & |a|=|b|, \\
\sqrt{b^{2}-a^{2}}, & |a|<|b| .\end{cases}
\end{gathered}
$$

Complex number argument

The value $\varphi=\operatorname{Arg} z$ is called the argument of the complex number $z$ :

$$
\begin{gathered}
\varphi=\arctan \frac{b}{a} ; \\
\varphi=\frac{b}{a}, a \neq 0 ; \\
\varphi= \begin{cases}\operatorname{artanh} \frac{b}{a}, & |a|>|b|, \\
\operatorname{artanh} \frac{a}{b}, & |a|<|b| .\end{cases}
\end{gathered}
$$

\subsubsection{Exponential representation}

The exponential representation is related to the trigonometric representation by Euler's formula. When writing arithmetic operations, these representations are interchangeable.

$$
\begin{gathered}
z=r \exp (\mathrm{i} \varphi) ; \\
z=r \exp (\varepsilon \varphi) ; \\
z= \begin{cases}r \exp (\operatorname{e} \varphi), & |a|>|b|, \\
0, & |a|=|b|, \\
\operatorname{er} \exp (\operatorname{ee} \varphi), & |a|<|b| .\end{cases}
\end{gathered}
$$

The modulus and argument of a complex number are specified in the same way as for trigonometric representation. 
6.2. Basic operations with complex numbers in different representations

6.2.1. Addition

For additive operations, we will use the algebraic representation of complex numbers. Let's set two complex numbers $z_{1}$ and $z_{2}$, then we get:

$$
\begin{aligned}
& z_{1}+z_{2}=(a+\mathrm{i} b)+(c+\mathrm{i} d)=(a+c)+\mathrm{i}(b+d) \\
& z_{1}+z_{2}=(a+\varepsilon b)+(c+\varepsilon d)=(a+c)+\varepsilon(b+d) \\
& z_{1}+z_{2}=(a+\mathrm{e} b)+(c+\mathrm{e} d)=(a+c)+\mathrm{e}(b+d) .
\end{aligned}
$$

\subsubsection{Subtraction}

Similarly, we write down for the subtraction of complex numbers $z_{1}$ and $z_{2}$ :

$$
\begin{aligned}
& z_{1}-z_{2}=(a+\mathrm{i} b)-(c+\mathrm{i} d)=(a-c)+\mathrm{i}(b-d) \\
& z_{1}-z_{2}=(a+\varepsilon b)-(c+\varepsilon d)=(a-c)+\varepsilon(b-d) \\
& z_{1}-z_{2}=(a+\mathrm{e} b)-(c+\mathrm{e} d)=(a-c)+\mathrm{e}(b-d) .
\end{aligned}
$$

\subsubsection{Multiplication (algebraic representation)}

For multiplication, it is more convenient to use exponential or trigonometric representations, however, with a small number of operands, the algebraic representation is also quite applicable.

$$
\begin{gathered}
z_{1} z_{2}=(a+\mathrm{i} b)(c+\mathrm{i} d)=(a c-b d)+\mathrm{i}(b c+a d) \\
z_{1} z_{2}=(a+\varepsilon b)(c+\varepsilon d)=a c+\varepsilon(b c+a d) \\
z_{1} z_{2}=(a+\mathrm{e} b)(c+\mathrm{e} d)=(a c+b d)+\mathrm{e}(b c+a d)
\end{gathered}
$$

\subsubsection{Multiplication (exponential representation)}

In the exponential representation, multiplication is reduced to the product of moduli and the sum of the arguments of complex factors.

$$
\begin{aligned}
& z_{1} z_{2}=r_{1} \exp \left(\mathrm{i} \varphi_{1}\right) r_{2} \exp \left(\mathrm{i} \varphi_{2}\right)=r_{1} r_{2} \exp \left(\mathrm{i}\left[\varphi_{1}+\varphi_{2}\right]\right) ; \\
& z_{1} z_{2}=r_{1} \exp \left(\varepsilon \varphi_{1}\right) r_{2} \exp \left(\varepsilon \varphi_{2}\right)=r_{1} r_{2} \exp \left(\varepsilon\left[\varphi_{1}+\varphi_{2}\right]\right) ; \\
& z_{1} z_{2}=r_{1} \exp \left(\operatorname{e} \varphi_{1}\right) r_{2} \exp \left(\mathrm{e} \varphi_{2}\right)=r_{1} r_{2} \exp \left(\mathrm{e}\left[\varphi_{1}+\varphi_{2}\right]\right) .
\end{aligned}
$$

\subsubsection{Multiplication (trigonometric representation)}

Multiplication in trigonometric representation does not differ from the multiplication in exponential representation (product of modules and sum of arguments of complex factors).

$$
\begin{gathered}
z_{1} z_{2}=r_{1}\left(\cos \varphi_{1}+\mathrm{i} \sin \varphi_{1}\right) r_{2}\left(\cos \varphi_{2}+\mathrm{i} \sin \varphi_{2}\right)=r_{1} r_{2}\left[\cos \left(\varphi_{1}+\varphi_{2}\right)+\mathrm{i} \sin \left(\varphi_{1}+\varphi_{2}\right)\right] \\
z_{1} z_{2}=r_{1}\left(1+\varepsilon \varphi_{1}\right) r_{2}\left(1+\varepsilon \varphi_{2}\right)=r_{1} r_{2}\left[1+\varepsilon\left(\varphi_{1}+\varphi_{2}\right)\right]
\end{gathered}
$$




$$
z_{1} z_{2}=\left\{\begin{array}{cc}
r_{1}\left(\cosh \varphi_{1}+\mathrm{e} \sinh \varphi_{1}\right) r_{2}\left(\cosh \varphi_{2}+\mathrm{e} \sinh \varphi_{2}\right) & \\
=r_{1} r_{2}\left[\cosh \left(\varphi_{1}+\varphi_{2}\right)+\mathrm{e} \sinh \left(\varphi_{1}+\varphi_{2}\right)\right], & |a|>|b|, \\
r_{1}\left(\sinh \varphi_{1}+\mathrm{e} \cosh \varphi_{1}\right) r_{2}\left(\sinh \varphi_{2}+\mathrm{e} \cosh \varphi_{2}\right) & \\
=r_{1} r_{2}\left[\cosh \left(\varphi_{1}+\varphi_{2}\right)+\mathrm{e} \sinh \left(\varphi_{1}+\varphi_{2}\right)\right], & |a|=|b|, \\
r_{1}\left(\cosh \varphi_{1}+\mathrm{e} \sinh \varphi_{1}\right) r_{2}\left(\sinh \varphi_{2}+\mathrm{e} \cosh \varphi_{2}\right) & \\
=r_{1} r_{2}\left[\sinh \left(\varphi_{1}+\varphi_{2}\right)+\operatorname{ecosh}\left(\varphi_{1}+\varphi_{2}\right)\right], & |a|<|b| .
\end{array}\right.
$$

The last expression looks somewhat cumbersome due to the form of Euler's formula for hyperbolic complex numbers.

\section{Relativistic operations}

To implement the mathematical apparatus of operations in the Minkowski space, it is proposed to use the corresponding system of complex numbers. Once again, note that for simplicity of description, we consider only one-dimensional motions, so that they can be described using complex numbers. When describing the full Minkowski space, it will be necessary to move from complex numbers to more complex objects, for example, to the corresponding type of quaternions [23-26]. In addition, it is known that relativistic calculations are generally non-commutative $[27,28]$.

The hyperbolic complex number $z=r \exp \{\mathrm{e} \varphi\}$ corresponds to a point in the Minkowski space. The argument $\varphi$ of the complex number $z$ is the angle between the tangent to the particle's world line and the time axis in the base frame. The argument is related to speed by the following: relationship:

$$
\varphi=\operatorname{artanh} \frac{v}{c} .
$$

It also has the name rapidity $[29,30]$.

The sequence of actions is as follows (we will consider only time-like intervals):

- As part of the operational part of preparing the system, the usual values are converted into the form of hyperbolic complex numbers ${ }^{+} \mathbb{C}$. Since the speeds are converted to rotations in the time plane (boosts), the following operation must be performed:

$$
\frac{v}{c} \rightarrow \tanh \varphi,
$$

that is, we get the corresponding complex number:

$$
z=\exp \left\{\operatorname{eartanh} \frac{v}{c}\right\}
$$

Here $\varphi$ is the argument of the corresponding complex number, and we neglect the module of the complex number.

- In the theoretical part, we perform calculations on the resulting complex numbers.

- Within the framework of the measuring operational part, we convert expressions in hyperbolic complex numbers ${ }^{+} \mathbb{C}$ into expressions in real numbers describing relativistic relations $\Lambda$ :

$$
\varphi \rightarrow \operatorname{artanh} \frac{v}{c} \text {. }
$$

The corresponding relativistic velocity will be:

$$
v_{\Lambda}=c \tanh \varphi
$$

For convenience, within the framework of one-dimensional motions, we can introduce the symbol of Einstein operations $\mathfrak{E}$, which directly transforms the operation $\mathrm{Op}_{\mathrm{Gal}}$ in the Galilean space into the operation $\mathrm{Op}_{\Lambda}$ in the Lorentz space:

$$
O \mathrm{p}_{\Lambda}=\mathfrak{E}\left(\mathrm{Op}_{\mathrm{Gal}}\right) .
$$


This operation masks the full cycle of transition from non-relativistic expressions Gal to relativistic $\Lambda$ by using hyperbolic complex numbers. We may write this transition in the form of a commutative diagram, which will have the following form:

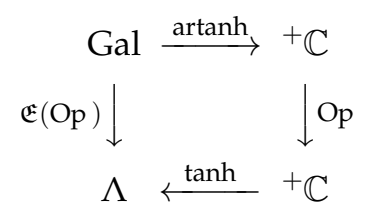

\section{Basic algebraic operations}

Let's write down the main operations.

\subsection{Addition of speeds}

Let's demonstrate the addition operation using both branches of diagrams (43), that is, both performing a full transformation cycle and performing a short relativistic transformation $\mathfrak{E}$. For other operations, for brevity, we will only use the $\mathfrak{E}$ operation.

The Lorentz transformation is determined by multiplying by a hyperbolic complex number with unit module $\exp \{\operatorname{e} \psi\}$, as a result, the Minkowski plane is rotated by the angle $\psi$ :

$$
z_{1}(\varphi) z_{2}(\psi)=\exp \{\operatorname{e} \varphi\} \exp \{\operatorname{e} \psi\}=\exp \{\operatorname{e}(\varphi+\psi)\}=z(\varphi+\psi)
$$

Replacing rapidity with speed, from (44) we get:

$$
z\left(\operatorname{artanh} \frac{v_{1}}{c}+\operatorname{artanh} \frac{v_{2}}{c}\right)=\exp \left\{\mathrm{e}\left(\operatorname{artanh} \frac{v_{1}}{c}+\operatorname{artanh} \frac{v_{2}}{c}\right)\right\} .
$$

Following the expression (7), let's pass on to real relativistic velocities:

$$
\left(v_{1}+v_{2}\right)_{\Lambda}=c \tanh \left(\operatorname{artanh} \frac{v_{1}}{c}+\operatorname{artanh} \frac{v_{2}}{c}\right) .
$$

Let's demonstrate the same with the E operation. Based on the diagram (43), we may write the addition operation:

$$
\begin{aligned}
\mathfrak{E}\left(v_{1}+v_{2}\right)=c \tanh (\operatorname{artanh} & \left.\frac{v_{1}}{c}+\operatorname{artanh} \frac{v_{2}}{c}\right)= \\
& =c \frac{\tanh \operatorname{artanh} \frac{v_{1}}{c}+\tanh \operatorname{artanh} \frac{v_{2}}{c}}{1+\tanh \operatorname{artanh} \frac{v_{1}}{c} \tanh \operatorname{artanh} \frac{v_{2}}{c}}=\frac{v_{1}+v_{2}}{1+\frac{v_{1} v_{2}}{c^{2}}} .
\end{aligned}
$$

We may write this operation for an arbitrary number of operands. For example, for three operands, we will get the following expression:

$$
\mathfrak{E}\left(v_{1}+v_{2}+v_{3}\right)=\frac{v_{1}+v_{2}+v_{3}+\frac{v_{1} v_{2} v_{3}}{c^{2}}}{1+\frac{v_{1} v_{2}+v_{1} v_{3}+v_{2} v_{3}}{c^{2}}} .
$$

It can be seen from the above relations that the addition operation in the proposed formalism coincides with the generally accepted one, but at the same time, it is easier to use.

\subsection{Multiplication of velocities}

The operation of multiplication of velocities is usually not used in relativistic calculations. 


$$
\begin{aligned}
& \mathfrak{E}\left(v_{1} v_{2}\right)=c \tanh \left(\operatorname{artanh} \frac{v_{1}}{c} \operatorname{artanh} \frac{v_{2}}{c}\right)= \\
&=c \tanh \left(\frac{1}{2} \ln \left(\frac{1+v_{1} / c}{1-v_{1} / c}\right) \frac{1}{2} \ln \left(\frac{1+v_{2} / c}{1-v_{2} / c}\right)\right)= \\
&=c \frac{\exp \left[\frac{1}{2} \ln \left(\frac{1+v_{1} / c}{1-v_{1} / c}\right) \ln \left(\frac{1+v_{2} / c}{1-v_{2} / c}\right)\right]-1}{\exp \left[\frac{1}{2} \ln \left(\frac{1+v_{1} / c}{1-v_{1} / c}\right) \ln \left(\frac{1+v_{2} / c}{1-v_{2} / c}\right)\right]+1} .
\end{aligned}
$$

\subsection{Multiplication by a number}

Consider the multiplication of the velocity vector by the number $k \in \mathbb{R}$ in the proposed representation:

$$
\begin{aligned}
& \mathfrak{E}(k v)=c \tanh \left(k \operatorname{artanh} \frac{v}{c}\right) \\
& \frac{\exp \left[k \ln \left(\frac{1+v / c}{1-v / c}\right)\right]-1}{\exp \left[k \ln \left(\frac{1+v / c}{1-v / c}\right)\right]+1}=c \frac{\left(\frac{1+v / c}{1-v / c}\right)^{k}-1}{\left(\frac{1+v / c}{1-v / c}\right)^{k}+1} \\
& c \frac{\left(1+\frac{v}{c}\right)^{k}-\left(1-\frac{v}{c}\right)^{k}}{\left(1+\frac{v}{c}\right)^{k}+\left(1-\frac{v}{c}\right)^{k}}
\end{aligned}
$$

Obviously, the expression (47) will never exceed the speed of light $c$ :

$$
\lim _{k \rightarrow \infty} \mathfrak{E}(k v)=c .
$$

As an example, we may write the expression for $\mathfrak{E}(2 v)$ using (45) and (47). From (45) we get:

$$
\mathfrak{E}(2 v)=\mathfrak{E}(v+v)=\frac{2 v}{1+\frac{v^{2}}{c^{2}}} .
$$

From (47) we obtain:

$$
\mathfrak{E}(2 v)=c \frac{\left(1+\frac{v}{c}\right)^{2}-\left(1-\frac{v}{c}\right)^{2}}{\left(1+\frac{v}{c}\right)^{2}+\left(1-\frac{v}{c}\right)^{2}}=c \frac{4 \frac{v}{c}}{2\left(1+\frac{v^{2}}{c^{2}}\right)}=\frac{2 v}{1+\frac{v^{2}}{c^{2}}} .
$$

We may see that the results of (48) and (49) are the same. Thus, the proposed representation does not contradict the speed addition procedure. The procedure for multiplying the speed by a number is consistent with the procedure for adding speeds.

Similarly, we can write the expression for $\mathfrak{E}(3 v)$ using (46) and (47). From (46) we obtain:

$$
\mathfrak{E}(3 v)=\mathfrak{E}(v+v+v)=\frac{3 v+\frac{v^{3}}{c^{2}}}{1+3 \frac{v^{2}}{c^{2}}} .
$$

From (47) we get:

$$
\mathfrak{E}(3 v)=c \frac{\left(1+\frac{v}{c}\right)^{3}-\left(1-\frac{v}{c}\right)^{3}}{\left(1+\frac{v}{c}\right)^{3}+\left(1-\frac{v}{c}\right)^{3}}=\frac{3 v+\frac{v^{3}}{c^{2}}}{1+3 \frac{v^{2}}{c^{2}}} .
$$

Obviously, the results of (50) and (51) are the same. 


\section{Repeal of paradoxes arising from relativistic operations}

Section 4 describes several so-called paradoxes, which lead to the fact that the operations of multiplication and division or multiplication by a number formally lead to the fact that speeds higher than the speed of light arise. Let's apply to these examples the obtained formula (47) for multiplication by a number.

\subsection{Oblique incidence of light}

Based on the equation (47), we can rewrite the paradoxical relation (1) in the relativistic case in the proposed representation:

$$
v=\mathfrak{E}\left(\frac{c}{\sin \varphi}\right)=c \frac{\left(1+\frac{c}{c}\right)^{1 / \sin \varphi}-\left(1-\frac{c}{c}\right)^{1 / \sin \varphi}}{\left(1+\frac{c}{c}\right)^{1 / \sin \varphi}+\left(1-\frac{c}{c}\right)^{1 / \sin \varphi}}=c .
$$

\subsection{Phase velocity}

Based on the generally accepted definition of multiplication by a number, from the equation (2) we concluded that the phase velocity might exceed the speed of light. However, if we replace the usual multiplication with the relativistic one according to the formula (47), we obtain:

$$
v_{p}(\varphi)=\mathfrak{E}\left(\frac{v_{p}(0)}{\cos \varphi}\right)=c \frac{\left(1+\frac{v_{p}(0)}{c}\right)^{1 / \cos \varphi}-\left(1-\frac{v_{p}(0)}{c}\right)^{1 / \cos \varphi}}{\left(1+\frac{v_{p}(0)}{c}\right)^{1 / \cos \varphi}+\left(1-\frac{v_{p}(0)}{c}\right)^{1 / \cos \varphi}} .
$$

Let's write the limit of the equation (52):

$$
\lim _{\cos \varphi \rightarrow 0} v_{p}(\varphi)=c .
$$

The phase speed is not greater than the speed of light.

Thus, with the consistent application of the proposed relativistic operations, no paradoxes arise.

\section{Conclusion}

The restriction of relativistic operations to only group operations seems to be insufficient. Insufficiency of these operations leads to paradoxes based on multiplication by a large number (division by a small number). The proposed algebraic extension of relativistic operations for velocities demonstrates that the considered class of paradoxes turns out to be imaginary. Instead of constructing a new relativistic algebra, the authors use the algebra of hyperbolic complex numbers. This approach is based on the Cayley - Klein classification and allows one to describe any space of a given model. As an advantage of this method, we can point out the fact that this approach can be extended from operations on collinear speeds to the full four-dimensional case with operations on 4-speeds using well-known mathematical procedures.

Author Contributions: Conceptualization, Dmitry S. Kulyabov and Leonid A. Sevastianov; writing—original draft preparation, Dmitry S. Kulyabov; writing—review and editing, Leonid A. Sevastianov and Anna V. Korolkova; visualization, Anna V. Korolkova; supervision, Leonid A. Sevastianov. All authors have read and agreed to the published version of the manuscript.

Funding: This paper has been supported by the RUDN University Strategic Academic Leadership Program.

Conflicts of Interest: The authors declare no conflict of interest. 


\section{References}

1. Ungar, A.A. Einstein's velocity addition law and its hyperbolic geometry. Computers and Mathematics with Applications 2007, 53, 1228-1250. doi:10.1016/j.camwa.2006.05.028.

2. Baker, G.A. Einstein Numbers. The American Mathematical Monthly 1954, 61, 39. doi:10.2307/2306894.

3. Vermeer, J. A geometric interpretation of Ungar's addition and of gyration in the hyperbolic plane. Topology and its Applications 2005, 152, 226-242. doi:10.1016/j.topol.2004.10.012.

4. Klein, F. Ueber die sogenannte Nicht-Euklidische Geometrie. In Gauß und die Anfänge der nicht-euklidischen Geometrie; SpringerVerlag Wien: Wien, 1985; Vol. 4, Teubner-Archiv zur Mathematik, pp. 224-238. doi:10.1007/978-3-7091-9511-6_5.

5. Yaglom, I.M.; Rozenfel'd, B.A.; Yasinskaya, E.U. Projective Metrics. Russian Mathematical Surveys 1964, $19,49-107$. doi:10.1070/RM1964v019n05ABEH001159.

6. Yaglom, I.M. Complex Numbers in Geometry; Academic Press, 1968.

7. Rozenfel'd, B.A.; Yaglom, I.M. On the geometries of the simplest algebras. Mat. Sbornik N. S. 1951, $28(70), 205-216$.

8. Kulyabov, D.S.; Korolkova, A.V.; Gevorkyan, M.N. Hyperbolic numbers as Einstein numbers. Journal of Physics: Conference Series 2020, 1557, 012027.1-5. doi:10.1088/1742-6596/1557/1/012027.

9. Gevorkyan, M.N.; Korolkova, A.V.; Kulyabov, D.S. Approaches to the implementation of generalized complex numbers in the Julia language. Workshop on information technology and scientific computing in the framework of the X International Conference Information and Telecommunication Technologies and Mathematical Modeling of High-Tech Systems (ITTMM-2020); Kulyabov, D.S.; Samouylov, K.E.; Sevastianov, L.A., Eds.; , 2020; Vol. 2639, CEUR Workshop Proceedings, pp. 141-157, [2007.09737].

10. Elnstein, A.; Infeld, L. The Evolution Of Physics; Cambridge University Press, 1938.

11. Minkowski, H. Raum und Zeit. Jahresbericht der Deutschen Mathematiker-Vereinigung 1909, 18, 75-88.

12. Penrose, R.; Rindler, W. Spinors and Space-Time; Vol. 1, Cambridge University Press, 1987. doi:10.1017/CBO9780511564048.

13. Dickson, L.E. On Quaternions and Their Generalization and the History of the Eight Square Theorem. The Annals of Mathematics 1919, 20, 155-171. doi:10.2307/1967865.

14. Heaviside, O. Electromagnetic Theory; Vol. 3, The Electrician: London, 1912.

15. Bolotovskiü, B.M.; Ginzburg, V.L. The Vavilov-Cerenkov Effect and the Doppler Effect in the Motion of Sources with Superluminal Velocity in Vacuum. Soviet Physics Uspekhi 1972, 15, 184-192. doi:10.1070/PU1972v015n02ABEH004962.

16. Bolotovskiǔ, B.M.; Serov, A.V. Radiation of superluminal sources in empty space. Physics-Uspekhi 2005, 48, 903-915. doi:10.1070/PU2005v048n09ABEH002568.

17. Barsukov, K.A.; Popov, V.N. On superluminal light spots. Physics-Uspekhi 1996, 39, 1181-1188. doi:10.1070/PU1996v039n11ABEH000182.

18. Sommerfeld, A.J.W. Ein Einwand gegen die Relativtheorie der Elektrodynamik und seine Beseitigung 1907. 8, 841-842.

19. Cayley, A. IV. A sixth memoir upon quantics. Philosophical Transactions of the Royal Society of London 1859, 149, 61-90. doi:10.1098/rstl.1859.0004.

20. Yaglom, I.M. A simple non-Euclidean geometry and its physical basis; Springer-Verlag, 1979.

21. Fjelstad, P. Extending special relativity via the perplex numbers. American Journal of Physics 1986, 54, 416-422. doi:10.1119/1.14605.

22. Band, W. Comments on Extending relativity via the perplex numbers. American Journal of Physics 1988, 56, 469-469. doi:10.1119/1.15582.

23. Buchheim, A. A Memoir on Biquaternions. American Journal of Mathematics 1885, 7, 293-326. doi:10.2307/2369176.

24. Silberstein, L. Quaternionic form of relativity. The London, Edinburgh, and Dublin Philosophical Magazine and Journal of Science, Series 6 1912, 23, 790-809. doi:10.1080/14786440508637276.

25. Girard, P.R. The quaternion group and modern physics. European Journal of Physics 1984, 5, 25-32. doi:10.1088/0143-0807/5/1/007.

26. Yefremov, A.P. Quaternions and Biquaternions: Algebra, Geometry and Physical Theories, 2005, [arXiv:math-ph/0501055].

27. Sommerfeld, A.J.W. On the addition of velocities in the theory of relativity. Uspekhi Fizicheskih Nauk 2010, $180,970$. doi:10.3367/UFNr.0180.201009e.0970.

28. Malykin, G.B. Noncommutative nature of the addition of noncollinear velocities in special relativity and the geometric phase method (commemorating the publication centennial of A Sommerfeld's work). Physics-Uspekhi 2010, 53, 923-927. doi:10.3367/UFNe.0180.201009d.0965.

29. Varičak, V. Anwendung der Lobatschefskijschen Geometrie in der Relativtheorie 1910. 11, 93-96.

30. Sommerfeld, A.J.W. Über die Zusammensetzung der Geschwindigkeiten in der Relativtheorie 1909. 10, 826-829. 